\title{
Evolución de las metas académicas en función del sexo y la edad y su influencia en el rendimiento académico en adolescentes murcianos
}

\section{Evolution of academic goals based on gender and age and their influence on academic achievement in adolescents in Murcia}

\author{
Cecilia Ruiz-Esteban ${ }^{1}$ \\ cruiz@um.es \\ InMaCulada MÉndeZ \\ inmamendez@um.es \\ Ángela Díaz-Herrero \\ adiaz@um.es \\ Universidad de Murcia, España.
}

\section{Resumen:}

Las metas académicas constituyen una de las variables más importantes desde el punto de vista motivacional para explicar las diferentes razones por las que los estudiantes se implican en las tareas y actividades de aprendizaje. En este estudio se analiza la evolución de las metas académicas y la influencia de éstas en el rendimiento a lo largo de los seis años que dura la enseñanza secundaria en función de variables sociodemográficas (sexo, edad y curso académico) en una muestra de 546 adolescentes murcianos. El $67.9 \%$ estaban cursando estudios de Enseñanza Secundaria Obligatoria y el $55.3 \%$ de los sujetos eran mujeres. Se administró el cuestionario CEMA y se recogieron las calificacio-

\begin{abstract}
:
Academic goals are one of the most important motivational variables to explain the different reasons whereby students get involved in tasks and learning activities. This study analyses gender and grade differences in academic goals together with the evolution of academic goals and their influence on academic achievement over the six years comprised within secondary education. The sample consisted of 546 students from Murcia. $67.9 \%$ were compulsory secondary education students and $53.3 \%$ were women. The Achievement Goals Tendencies Questionnaire (AGTQ) was administered and average grades were used as achievement data. The results revealed that academic goals varied
\end{abstract}

1 Dirección para correspondencia (correspondence address):

Cecilia Ruiz Esteban. Departamento de Psicología Evolutiva y de la Educación. Facultad de Educación. Universidad de Murcia. Campus de Espinardo, s/n. 30100 Espinardo (España). 
Evolución de las metas académicas en función del sexo y la edad y su influencia en el rendimiento académico en adolescentes murcianos

Cecilia Ruiz-Esteban, Inmaculada Méndez y Ángela Díaz-Herrero

nes medias como variable de rendimiento. Los resultados mostraban diferentes patrones motivacionales por sexo, siendo las chicas las que mostraban una orientación motivacional mayor hacia el logro. Por curso académico, se observó que la orientación hacia metas de refuerzo social aumentaba a medida que se avanzaba en éstos, sin embargo no se pudo concluir que haya un patrón evolutivo estable para la motivación de logro. Los resultados académicos fueron una variable predictora de los patrones motivacionales intrínsecos (metas de orientación hacia el aprendizaje y metas de logro) mientras que la edad y el curso académico lo fueron para patrones motivacionales extrínsecos. Este estudio sugiere que sus resultados podrían utilizarse para diseñar nuevos instrumentos de evaluación y poder generar programas de intervención psicoeducativa para la mejora de la motivación y fomento del estudio en estudiantes de secundaria.

\section{Palabras clave:}

Metas de aprendizaje; sexo; rendimiento académico; educación secundaria. significantly according to gender and that women presented a significantly higher motivational orientation towards achievement goals. In terms of academic level, it is observed that the orientation toward social reinforcement goals increases the higher the academic year is, but we cannot conclude that there is a stable pattern for achievement motivation. Academic achievement was a predictor of intrinsic motivational patterns (goals orientation toward learning and achievement goals), while age and academic year were so for extrinsic motivational patterns. This study suggests that its results could be used to design new evaluation tools and to generate psychoeducational intervention programs to improve motivation and foster study in high school students.

\section{Key words:}

Academic goals; gender; academic achievement; secondary education.

\section{Résumé:}

Les objectifs académiques sont l'une des variables les plus importantes du point de vue motivationnel pour expliquer les différentes raisons pour lesquelles les élèves sont impliqués dans des tâches et des activités d'apprentissage. Dans cette étude, on analyse l'évolution des objectifs académiques et l'influence de ceux-ci dans les résultats durant les 6 ans que dure l'enseignement secondaire en fonction de variables sociodémographiques (sexe, âge et cours) pour un échantillon composé de 546 collégiens et lycéens murciens. $67.9 \%$ de ces élèves faisaient des études d'enseignement secondaire et $55.3 \%$ d'entre eux étaient des femmes. On leur a fait passer le questionnaire CEMA et on a pris en compte les qualifications moyennes comme variable de performance. Les résultats montrent les différentes tendances de motivation qu'il existe par sexe, les filles montrant une claire motivation vers l'obtention d'un but. On observe aussi que selon I'âge, l'orientation vers des buts de renforcement social augmente en même temps que les années académiques, on ne peut cependant pas en conclure qu'il existe une tendance évolutive stable quant à la motivation vers la réussite. Les résultats académiques ont été une variable qui a servi à prédire les tendances de motivation intrinsèques (buts d'orientation vers I'apprentissage et buts d'accomplissement) alors que l'âge et l'année académique ont été des variables pour des tendances de motivation extrinsèque. Cette étude suggère que les résultats pourraient être utilisés pour concevoir de nouveaux outils d'évaluation et pour générer des programmes d'intervention psychopédagogique afin d'améliorer la motivation et l'encouragement de l'étude chez les élèves du secondaire.

\section{Mots clés:}

Objectifs d'apprentissage; sexe; résultats académiques; I'enseignement secondaire. 
Fecha de recepción: 25-7-2016

Fecha de aceptación: 1-3-2017

\section{Introducción}

Las metas académicas constituyen una de las variables más importantes desde el punto de vista motivacional para explicar las diferentes razones por las que los estudiantes se implican en las tareas y actividades de aprendizaje. Al mismo tiempo, están relacionadas con su rendimiento académico. Las metas académicas que persigue el estudiante organizan y regulan su comportamiento de cara a la consecución de un determinado logro (Valle et al., 2009).

Diversos autores han estudiado las orientaciones hacia las metas académicas y han establecido una clasificación de las mismas. Aunque encontramos algunas diferencias entre los autores, podemos concluir que hay un cierto consenso en señalar dos orientaciones básicas hacia la meta: la primera se orienta hacia el aprendizaje y la comprensión, mientras que la segunda se centra en el Yo y el rendimiento (Gaeta, 2011). Es decir, mientras algunos estudiantes se involucran en las actividades académicas para mejorar sus capacidades y aprender (orientación al aprendizaje), otros se orientan hacia la consecución de metas extrínsecas como la obtención de buenas notas, recompensas, juicios positivos de los demás y evitación de todo tipo de valoraciones negativas (orientación al rendimiento) (Dweck, 1986; Elliot y Dweck, 1988). Otros autores (Alonso, 1991; Alonso y Montero, 1992; Núñez et al., 1997) proponen cuatro categorías para agrupar las metas que los estudiantes persiguen y que determinan su modo de afrontar las actividades académicas: (a) las metas relacionadas con la tarea, que se asocian con el interés por desarrollar o mejorar sus capacidades y el aprendizaje; (b) las metas relacionadas con la autovaloración (orientación al Yo), que expresan la búsqueda de experimentar el orgullo y satisfacción que sigue al éxito o el evitar la "vergüenza" que acompaña al fracaso; (c) las metas orientadas a la valoración social, que expresan la motivación ante las tareas académicas encaminadas hacia la valoración y aprobación de otros, y (d) las metas relacionadas con la consecución de recompensas externas, que se relacionan tanto con la consecución de premios o recompensas como con la evitación de todo lo que im- 
Evolución de las metas académicas en función del sexo y la edad y su influencia en el rendimiento académico en adolescentes murcianos

Cecilia Ruiz-Esteban, Inmaculada Méndez y Ángela Díaz-Herrero

plique pérdida de situaciones u objetos valorados por la persona. Sin embargo, Harackiewicz, Barron, Pintrich, Elliot y Thrash (2002) consideran que esta concepción es más compleja ya que los estudiantes no persiguen una única meta sino que pueden trabajar considerando diferentes orientaciones en función de sus características personales, de la propia tarea y/o del contexto.

Un amplio número de investigaciones han puesto de manifiesto la existencia de distintos patrones motivacionales entre los estudiantes de secundaria en relación al rendimiento. En este sentido, Meece, Anderman y Anderman, (2006) señalan que los centros educativos que se focalizan en incrementar su rendimiento reforzando la competencia cognitiva y la competitividad provocan una bajada de la motivación, y Steinmayr y Spinarth, (2009) indican que la motivación es un predictor del rendimiento tan potente al menos como la inteligencia. Entre estos patrones se señala como variable determinante las orientaciones hacia las metas académicas que persigue el sujeto. Una de las razones por las que el alumnado se implica en las tareas y actividades de aprendizaje tiene que ver con los tipos de metas académicas que los estudiantes exhiben (Rodríguez et al., 2001) ya que se las considera predictores importantes de un gran número de procesos de logro y desempeño. Por ejemplo, Valle et al. (2007) manifiestan que los alumnos orientados a metas con patrones motivacionales intrínsecos exhiben mejores niveles de rendimiento académico mientras que aquellos con metas orientadas hacia el YO muestran un rendimiento más bajo

En la adolescencia, estos patrones motivacionales difieren en función del sexo (Delgado, Inglés, García-Fernández, Castejón y Valle, 2010; Pintrich y Schunk, 2002; Shibley y Durik, 2005) ya que influyen en el modo de afrontar una tarea, encontrándose en diversos trabajos una mayor motivación de logro por parte de las chicas (p.e. Shibley y Durik, 2005). También la edad parece ser una variable que interfiere en los patrones motivacionales (Delgado et al., 2010; Murphy y Alexander, 2000). Navas, González y Torregrosa (2002) encontraron que en la adolescencia la diferenciación que se obtiene con respecto a los sujetos de educación primaria no solo tiene que ver con los patrones evolutivos y madurativos sino también con los cambios de actuación del profesorado que a su vez, influyen y son influidos por los estudiantes. Las valoraciones sociales y las posibles repercusiones sociales de su conducta relacionada con sus aprendizajes académicos (profesión futura, posibilidades de elección, 
valoración social positiva) se convierten en claves para explicar el peso de las metas de refuerzo social en esta etapa evolutiva.

Así, esta investigación pretende analizar la evolución de las metas académicas y la influencia de éstas en el rendimiento a lo largo de los 6 años que dura la enseñanza secundaria en función de variables sociodemográficas (sexo, edad y curso académico).

\section{Marco empírico}

\section{Participantes}

Los participantes del estudio fueron 546 estudiantes murcianos. El 67.9\% estaban cursando estudios de Enseñanza Secundaria Obligatoria. De ellos, el $14.7 \%$ fueron estudiantes de primero $(n=80)$, el $20.9 \%$ de segundo $(n=114)$; el $18.5 \%$ de tercero $(n=118)$ y el $13.9 \%$ de cuarto $(n=79)$, mientras que el $32.1 \%$ restante estaban cursando bachiller (18.7\% primero). La media de edad fue de 15.08 años. El 55.3\% de los sujetos eran mujeres.

\section{Instrumento}

Se utilizó el CEMA traducido y validado por Núñez et al. (1997) al contexto español en estudiantes de educación secundaria, a partir del "Questionnaire to Measure Achievement Goal Tendencies" de Hayamizu y Weiner (1991).

El CEMA en su versión española está compuesto por 20 ítems y fue diseñado para medir tres tendencias u orientaciones de metas: Metas de Aprendizaje (MA), que indican la tendencia de los estudiantes por aprender con el propósito de adquirir conocimientos y aumentar su competencia -8 ítems-, Metas de Logro (ML), que reflejan la tendencia de los estudiantes por aprender con la intención de obtener buenas notas en los exámenes y avanzar en los estudios -6 ítems- $y$, Metas de Refuerzo Social (MRS), que indican la tendencia de los estudiantes por aprender con el propósito de obtener la aprobación social de padres y de profesores y evitar su rechazo -6 ítems-.

Las respuestas a cada uno de los ítems del cuestionario aparecen categorizadas en una escala tipo Likert que va desde 1 (nunca) hasta 5 (siempre). 
Evolución de las metas académicas en función del sexo y la edad y su influencia en el rendimiento académico en adolescentes murcianos

Cecilia Ruiz-Esteban, Inmaculada Méndez y Ángela Díaz-Herrero

Las propiedades psicométricas del cuestionario han sido analizadas en España con estudiantes de primaria (García et al., 1998; Jover, Navas y Sampascual, 2008; Navas et al., 2002), secundaria (García et al., 1998; Navas et al., 2002) y universidad (Navas et al., 2002). Además, la fiabilidad y validez del CEMA han sido adecuadas en alumnos de secundaria japoneses (Hayamizu, Ito y Yoshizaki, 1989), así como en estudiantes universitarios de Estados Unidos (Hayamizu y Weiner, 1991), Francia (Duyperat y Escribe, 2000), Perú (Escurra et al., 2005), Argentina (Corral de Zurita, 2003) y México (Gaeta, Cavazos, Sánchez, Rosário y Högemann, 2015).

El análisis factorial exploratorio realizado por los autores originales (Hayamizu y Weiner, 1991) reveló la existencia de tres factores: MA, MRS y ML que explicaron el $52.4 \%$ de varianza total.

Los índices de fiabilidad (alfa de Cronbach) del instrumento original (Núñez et al., 1997) oscilan desde $\alpha=.75$ para el factor metas de evitación del trabajo para una defensa del Yo hasta $\alpha=.87$ para el factor metas centradas en el interés por la materia. La consistencia interna del cuestionario resultó adecuada con unos coeficientes moderados de fiabilidad (alfa de Cronbach) para las escalas MA (.89), ML (.71) y MRS (.78). En nuestro país, García et al. (1998) y Navas et al. (2002) obtuvieron valores de fiabilidad y validez similares a los autores originales, confirmando la adecuación de la prueba en adolescentes españoles

Del mismo modo, para recabar información sobre el rendimiento académico se solicitó al alumnado que indicasen la nota media que habían obtenido hasta el momento.

\section{Procedimiento}

Se entrevistó a los directivos de los centros educativos para exponer los objetivos de la investigación y solicitar su colaboración. Posteriormente se solicitó el consentimiento informado de todos los posibles participantes menores de 18 años, pidiendo autorización a los padres o tutores legales para que sus hijos pudieran participar en la investigación. Una vez identificados los estudiantes, la administración de la prueba se realizó de forma colectiva en el aula. El tiempo medio de aplicación del CEMA fue de 7 minutos. 


\section{Análisis de datos}

En primer lugar, se realizó una diferencia de medias (t de Student) para muestras independientes para determinar si existían diferencias por sexo en cada una de las escalas. Más tarde se utilizó un análisis de varianza (ANOVA) para determinar las diferencias por curso para cada una de las escalas. Posteriormente para identificar los cursos entre los que se encontraban las diferencias estadísticamente significativas se utilizaron comparaciones post-hoc mediante la prueba de Bonferroni. Se utilizó la prueba de Brown-Forsythe cuando no pudo ser admitida la igualdad de varianzas a través de la prueba de Levene. Finalmente, a través de los análisis de regresión jerárquicos se pudo contrastar la potencia predictiva de los grupos de variables independientes (variables sociodemográficas como la edad, el sexo y el curso así como académica a través de la nota media obtenida) con respecto a la variable dependiente bajo estudio (MA, MRS y ML). Se estimó oportuno la utilización del método "introducir" para analizar los efectos de cada bloque y por lo tanto, identificar los que mejor explican las modificaciones ocurridas en la variable dependiente.

Los análisis se realizaron con el SPSS v.19

\section{Resultados}

\section{Diferencias en función de variables sociodemográficas (sexo, edad y curso) en las metas académicas.}

En primer lugar, la tabla 1 muestra las diferencias de sexo en las escalas del CEMA. Los resultados señalan que sólo existían diferencias estadísticamente significativas en una de las escalas, las Metas de Logro (ML). La prueba t de Student mostró diferencias de medias significativas, asumiendo varianzas homogéneas, $T(544)=-2.132 ; p=.033$ con un efecto bajo-medio ( $d=.18)$. 
Evolución de las metas académicas en función del sexo y la edad y su influencia en el rendimiento académico en adolescentes murcianos

Cecilia Ruiz-Esteban, Inmaculada Méndez y Ángela Díaz-Herrero

Tabla 1. Diferencias de sexo en metas académicas.

\begin{tabular}{|c|c|c|c|c|}
\hline \multirow[t]{3}{*}{ METAS ACADÉMICAS } & \multicolumn{2}{|c|}{ SEXO } & \multirow[b]{3}{*}{ F de Levene } & \multirow[b]{3}{*}{$p$} \\
\hline & VARONES & MUJERES & & \\
\hline & $M(D T)$ & $M(D T)$ & & \\
\hline MA (8-40) & $32.05(4.87)$ & $32.43(4.53)$ & 0.74 & .34 \\
\hline MRS (6-30) & $20.90(5.81)$ & $20.11(5.72)$ & 0.23 & .10 \\
\hline ML (6-30) & $26.01(3.82)$ & $26.65(3.15)$ & 6.37 & $.03 *$ \\
\hline
\end{tabular}

Las adolescentes presentaron una orientación hacia las metas de logro significativamente más alta que la de los varones de la misma edad. No se encontró una orientación motivacional definida en los varones, mientras que las chicas mantuvieron una orientación hacia la consecución de buenos resultados académicos. Si bien se trata de una orientación extrínseca, sí podemos indicar que estaban más preocupadas que sus compañeros varones por progresar en sus estudios.

En segundo lugar, la tabla 2 nos indica las diferencias por curso académico en las escalas del CEMA en la prueba ANOVA de un factor.

Tabla 2. Diferencias por curso en orientación hacia las metas.

\begin{tabular}{|c|c|c|c|c|c|c|c|c|}
\hline \multirow{3}{*}{$\begin{array}{l}\text { METAS } \\
\text { ACADÉMICAS }\end{array}$} & \multicolumn{6}{|c|}{ CURSO } & & \\
\hline & $1^{\circ} \mathrm{E} . \mathrm{SO}$ & $2^{\circ} \mathrm{ESO}$ & $3^{\circ} \mathrm{ESO}$ & $4^{\circ} \mathrm{ESO}$ & $1^{\circ} \mathrm{BAC}$ & $2^{\circ} \mathrm{BAC}$ & & \\
\hline & $M(\mathrm{DT})$ & $M(\mathrm{DT})$ & $M(\mathrm{DT})$ & $M(\mathrm{DT})$ & $M(\mathrm{DT})$ & $M(\mathrm{DT})$ & $F$ & $p$ \\
\hline MA (8-40) & $\begin{array}{l}31.92 \\
(4.93)\end{array}$ & $\begin{array}{l}32.70 \\
(4.92)\end{array}$ & $\begin{array}{l}31.76 \\
(4.70)\end{array}$ & $\begin{array}{l}32.51 \\
(4.54)\end{array}$ & $\begin{array}{l}32.37 \\
(3.36)\end{array}$ & $\begin{array}{l}32.31 \\
(4.69)\end{array}$ & .97 & .43 \\
\hline MRS (6-30) & $\begin{array}{l}23.42 \\
(4.29)\end{array}$ & $\begin{array}{l}23.03 \\
(5.07)\end{array}$ & $\begin{array}{l}20.69 \\
(6.02)\end{array}$ & $\begin{array}{l}21.48 \\
(5.61)\end{array}$ & $\begin{array}{l}16.37 \\
(5.72)\end{array}$ & $\begin{array}{l}20.48 \\
(5.88)\end{array}$ & 15.72 & $.00^{*}$ \\
\hline ML (6-30) & $\begin{array}{l}27.80 \\
(1.91)\end{array}$ & $\begin{array}{l}27.26 \\
(3.07)\end{array}$ & $\begin{array}{l}26.05 \\
(3.52)\end{array}$ & $\begin{array}{l}27.48 \\
(2.45)\end{array}$ & $\begin{array}{l}26.22 \\
(4.03)\end{array}$ & $\begin{array}{l}25.98 \\
(2.72)\end{array}$ & 3.64 & $.00^{*}$ \\
\hline
\end{tabular}

$\overline{M A}=$ Metas de Aprendizaje, $M R S=$ Metas de Refuerzo Social, ML=Metas de Logro

La tabla 3 nos revela la existencia de diferencias estadísticamente significativas en dos de las tres escalas en que dividimos la orientación hacia las metas de los adolescentes. No encontramos diferencias estadísticamente significativas en la motivación intrínseca que se orientaría principalmente hacia la adquisición de conocimientos. Sin embargo, encontramos que el curso académico marcaba diferencias en cuanto al 
perfil motivacional extrínseco de los adolescentes. Es decir, en la orientación de su actividad académica hacia la obtención de juicios de aprobación y evitación del rechazo de sus progenitores y docentes así como a la obtención de buenas notas en los exámenes.

Las pruebas post-hoc indicaban que las orientaciones hacia MRS aumentaban a medida que aumentaban los cursos académicos. Sin embargo, no se obtuvo un patrón estable en ML puesto que las diferencias de medias significativas se obtuvieron únicamente entre segundo curso de la ESO y segundo de bachiller (véase tabla 3).

Tabla 3. Orientaciones hacia las metas en función del curso académico.

\begin{tabular}{llll}
\hline Variables & Curso académico & & \\
\cline { 2 - 4 } & $F$ & Diferencias de medias & $p$ \\
\hline Orientaciones & Brown-Forsythe & $1^{\circ} \mathrm{ESO}-1^{\circ} \mathrm{BAC}=3.25$ & $.00^{*}$ \\
hacia MRS & $F(5 ; 510.182)=15.910, p=.000$ & $1^{\circ} \mathrm{ESO}-2^{\circ} \mathrm{BAC}=5.21$ & $.00^{*}$ \\
& & $2^{\circ} \mathrm{ESO}-3^{\circ} \mathrm{ESO}=2.23$ & $.04^{*}$ \\
& $2^{\circ} \mathrm{ESO}-4^{\circ} \mathrm{ESO}=2.85$ & $.00^{*}$ \\
& $2^{\circ} \mathrm{ESO}-1^{\circ} \mathrm{BAC}=4.39$ & $.00^{*}$ \\
& & $2^{\circ} \mathrm{ESO}-2^{\circ} \mathrm{BAC}=6.35$ & $.00^{*}$ \\
& & $3^{\circ} \mathrm{ESO}-2^{\circ} \mathrm{BAC}=4.11$ & $.00^{*}$ \\
Orientaciones & $4^{\circ} \mathrm{ESO}-2^{\circ} \mathrm{BAC}=3.47$ & $.00^{*}$ \\
\hline
\end{tabular}

\section{Predicción de las metas académicas según variables sociodemográficas y académicas}

Finalmente, el análisis de regresión utilizando como criterio MA y como variables predictoras las relativas a variables sociodemográficas (curso, sexo y edad) y notas académicas, explicó el $8.3 \%$ de la varianza. Los coeficientes de regresión estandarizados Beta indicaron que, de todas las variables predictoras introducidas, resultó significativa únicamente la nota académica (Beta $=.306 ; t=6.876 ; \mathrm{p}=.000$ ).

Del mismo modo, el análisis de regresión utilizando como criterio MRS y como variables predictoras las relativas a curso, sexo, edad y notas académicas, explicó el $13.8 \%$ de la varianza. Los coeficientes de regresión estandarizados Beta indicaron que de todas las variables predictoras introducidas, resultaron significativas las siguientes: el curso (Beta=-.145; $t=-2.382 ; \mathrm{p}=.018$ ) y la edad (Beta $=-.217 ; t=-3.555 ; \mathrm{p}=.000$ ). 
Evolución de las metas académicas en función del sexo y la edad y su influencia en el rendimiento académico en adolescentes murcianos

Cecilia Ruiz-Esteban, Inmaculada Méndez y Ángela Díaz-Herrero

Asimismo, el análisis de regresión utilizando como criterio ML y como variables predictoras las relativas a curso, sexo, edad y notas académicas, explicó el $10.5 \%$ de la varianza. Los coeficientes de regresión estandarizados Beta indicaron que de todas las variables predictoras introducidas, resultó significativa la nota académica (Beta $=.329 ; t=7.471 ; \mathrm{p}=.000$ ).

\section{Conclusiones}

Esta investigación pretendía analizar la evolución de las metas académicas y la influencia de éstas en el rendimiento a lo largo de los seis años que dura la enseñanza secundaria en función de variables sociodemográficas (sexo, edad y curso académico).

Los resultados mostraron que las chicas presentaron una orientación hacia las metas de logro significativamente más alta que la de los varones de la misma edad. No se encontró una orientación motivacional definida en los varones, mientras que las chicas mantuvieron una orientación hacia la consecución de buenos resultados académicos. Estos datos no coinciden con los obtenidos por Dupeyrat y Escribe (2000) y Delgado et. al, (2010) sin embargo están en la línea de los hallazgos de Shibley y Durik (2005) que señalaban que la orientación hacia el logro cada vez mayor que mostraban las adolescentes podría explicarse por la apertura hacia nuevas oportunidades de educación como la realización de estudios superiores en las que además obtienen de media mejores puntuaciones que los varones.

Por otro lado, encontramos que el curso académico marcaba diferencias en cuanto al perfil motivacional extrínseco de los adolescentes. Es decir, en la orientación de su actividad académica hacia la obtención de juicios de aprobación y evitación del rechazo de sus progenitores y docentes así como a la obtención de buenas notas en los exámenes. Esta tendencia motivacional genera de una manera indirecta una mayor probabilidad de alcanzar un bajo rendimiento académico (Covington, 2000). Algunos autores señalan la necesidad de realizar intervenciones para mejorar estos patrones motivacionales (Martin, 2004).

La evolución de las orientaciones motivacionales también ha arrojado datos interesantes. La orientación hacia metas de refuerzo social aumentaba a medida que aumentaban los cursos académicos. Pese a que estos datos no corroboran los encontrados por Delgado et al, (2010), 
consideramos que la gran importancia que cobra el grupo de amigos en la adolescencia puede estar detrás de estos resultados. Navas et al. (2002) señalan que los adolescentes ya no buscan la aprobación de una autoridad superior, sino que intentan encontrar un sitio en la sociedad en la que viven. Tal y como señala Alonso (1991), parece haber una serie de metas que podrían considerarse más particulares de los adolescentes que están directamente relacionadas con la autovaloración con los patrones extraídos de la influencia social de su entorno, lo que explicaría la importancia de las metas de refuerzo social en esta etapa.

Sin embargo, no se obtuvo un patrón estable en la orientación hacia las metas de logro a lo largo de los cursos aunque sí se observan diferencias de medias significativas entre segundo curso de la ESO y segundo de bachiller, lo que está en la línea de lo observado por otros autores (Delgado et al. 2010; Valle, Cuevas y Núñez, 1997).

El análisis de las diferencias en la orientación hacia las metas puede proporcionar información acerca de distintas tendencias hacia el estudio que presenta el alumnado y por extensión mediatizan su rendimiento, la cual podría emplearse para diseñar nuevos instrumentos de evaluación y poder generar programas de intervención psicoeducativa para la mejora de la motivación y fomento del estudio en estudiantes de secundaria. Si bien es cierto que en los últimos años los orientadores se han vuelto imprescindibles en los centros de enseñanza secundaria, el amplio número de funciones que deben desempeñar, junto con el número de estudiantes hace complicado que puedan acometer programas de intervención encaminados a la mejora del rendimiento académico. Así, en la actualidad los programas de mejora del proceso de enseñanza-aprendizaje que refuercen la adquisición de competencias en estrategias de aprendizaje, la motivación o la atribución causal de los estudiantes han tenido que ser relegados a un segundo plano. Se necesitarían más recursos humanos y materiales para afrontar este tipo de tareas.

Los resultados académicos fueron una variable predictora de los patrones motivacionales intrínsecos (metas de orientación hacia el aprendizaje y metas de logro), mientras que la edad y el curso académico lo fueron para patrones motivacionales extrínsecos. No obstante, resulta indiscutible la relación entre rendimiento y patrón motivacional. Los especialistas más destacados en este tema como por ejemplo Paris, Lipson y Wixson (1983), Pintrich (1989), Pintrich y De Groot (1990) opinan que, para tener buenos resultados académicos, los alumnos necesitan poseer 
Evolución de las metas académicas en función del sexo y la edad y su influencia en el rendimiento académico en adolescentes murcianos

Cecilia Ruiz-Esteban, Inmaculada Méndez y Ángela Díaz-Herrero

tanto "voluntad " como "habilidad", lo que conduce a la necesidad de integrar ambos aspectos. Este trabajo se ha centrado en los aspectos motivacionales dejando de lado los cognitivos, lo que sin duda constituye una de las limitaciones de este estudio.

Resulta llamativo que los estudiantes con mejores resultados académicos se orienten hacia las metas de logro y no hacia las metas de aprendizaje como muestran otros estudios previos (Valle et al. 2007, 2008) La limitación de plazas de la mayoría de las titulaciones universitarias y la importancia de las calificaciones en el futuro académico de los adolescentes pueden pervertir sus patrones motivacionales. De manera análoga sucede con la alta competitividad generada por el sistema de becas. En definitiva, puede ser el propio sistema educativo universitario el que empuje a nuestros estudiantes adolescentes hacia una preocupación excesiva por la calificación en lugar de orientarlos hacia el aprendizaje como sería deseable.

Entre las limitaciones de este estudio hay que tener en cuenta que los resultados hay que considerarlos con las prevenciones propias de los estudios trasversales. Además, es difícil que podamos identificar estudiantes con orientaciones hacia una única meta, cuando lo habitual es encontrar estudiantes con orientación simultánea a más de una meta que puede modificarse, por ejemplo, en función de la tarea. Por otra parte, en este estudio sólo se han utilizado medidas de autoinforme que pueden estar influidas por la deseabilidad social. Dado además que el porcentaje de varianza explicado no es muy alto, sería deseable utilizar otros instrumentos de evaluación como la observación o la entrevista para poder triangular los hallazgos y establecer qué otras variables pueden estar influyendo en las metas académicas exhibidas por nuestros adolescentes.

\section{Referencias}

Alonso, J. (1991). Motivación y aprendizaje en el aula. Cómo enseñar a pensar. Madrid: Santillana.

Alonso, J. y Montero, Y. (1992). Motivación y aprendizaje escolar. En C. Coll, J. Palacios y A. Marchesi (compils.), Desarrollo psicológico y educación, II. Psicología de la Educación. Madrid: Alianza.

Covington, M. V. (2000). Goal theory, motivation, and school achievement: An integrative review. Annual Review of Psychology, 51(1), 171-200. 
Evolución de las metas académicas en función del sexo y la edad y su influencia en el rendimiento académico en adolescentes murcianos Cecilia Ruiz-Esteban, Inmaculada Méndez y Ángela Díaz-Herrero

Corral de Zurita, N. J. (2003). Metas académicas, atribuciones causales y rendimiento académico. Universidad Nacional del Nordeste. Comunicaciones científicas y tecnológicas. Recuperado de: http://www1.unne.edu.ar/cyt/2003/comunicaciones/09Educacion/D-006. pdf.

Delgado, B., Inglés, C. J., García-Fernández, M., Castejón, J. L. y Valle, A. (2010). Diferencias de género y curso en metas académicas en alumnos de Educación Secundaria Obligatoria. Revista Española de Pedagogía, 245, 67-84.

Dupeyrat, C. y Escribe, C. (2000). Orientations de but: Validation du questionnaire de Hayamizu et Weiner et relations avec les conceptions de I'intelligence. European Review of Applied Psychology, 50(1), 73-80.

Dweck, C.S. (1986). Motivational processesaffecting learning. American Psychologist, 41, 1040-1048.

Elliot, E.S. y Dweck, C.S. (1988). Goals: An approach to motivation and achievement. Journal of Personality and Social Psychology, 54, 5-12.

Escurra, L.M., Delgado, A., Guevara, G., Torres, M., Quezada, R., Morocho, J., Rivas, G. y Santos, J. (2005). Relación entre el autoconcepto de las competencias, las metas académicas y el rendimiento en alumnos universitarios de la ciudad de Lima. Revista de Investigación en Psicología, 8(1), 87-106.

Gaeta, M.L. (2011). La autorregulación del aprendizaje en la adolescencia: estrategias metacognitivas, motivacionales y emocionales. Saarbrüken, Alemania: Editorial Académica Española.

Gaeta, M. L., Cavazos, J., Sánchez, A. P., Rosário, P. y Högemann, J. (2015). Propiedades psicométricas de la versión mexicana del Cuestionario para la Evaluación de Metas Académicas (CEMA). Revista Latinoamericana de Psicología, 47(1), 16-24.

García, M.S., González-Pienda, J.A., Nuñez, J.C., González-Pumariega, S., Alvarez, L., Roces, C., González, R. y Valle, A. (1998). El cuestionario de metas académicas (C.M.A.): Un instrumento para la evaluación de la orientación motivacional de los alumnos de educación secundaria. Aula Abierta, 71, 175-199.

Harackiewicz, J., Barron, K., Pintrich, P., Elliot, A. y Thrash, T.(2002). Revision of achievement goal theory: Necessary and illuminating. Journal of Educational Psychology, 94, 638-645.

Hayamizu, T. y Weiner, B. (1991). A test Dweck's model of achievement goals as related to perceptions of ability. Journal of Experimental Education, 59, 226-234.

Hayamizu, T., Ito, A. y Yoshizaki, K. (1989).Cognitive motivational processes mediated by achievement goal tendencies. Japanese Psychological Research, 31, 179-189.

Jover, I., Navas, L. y Sampascual, G. (2008). Metas académicas en alumnos con ceguera y deficiencia visual. Revista Española de Pedagogía, 66(239), 49-64.

Martin, A. J. (2004). School motivation of boys and girls: Differences of degree, differences of kind, or both?. Australian Journal of Psychology, 56(3), 133-146.

Meece, J. L., Anderman, E. M. y Anderman, L. H. (2006). Classroom goal structure, student motivation, and academic achievement. Annual Review of Psychology, 57, 487503.

Murphy, P. K. y Alexander, P. A. (2000). A motivated exploration of motivation terminology. Contemporary Educational Psychology, 25(1), 3-53. 
Evolución de las metas académicas en función del sexo y la edad y su influencia en el rendimiento académico en adolescentes murcianos

Cecilia Ruiz-Esteban, Inmaculada Méndez y Ángela Díaz-Herrero

Navas, L., González, C. y Torregrosa, G. (2002) Metas de aprendizaje: Un análisis transversal de las estructuras factoriales que presentan. Revista de Psicología General y Aplicada, 55(4), 553-564.

Núñez, J., González-Pienda, J., González-Pumariega, S, García, M. y Roces, C. (1997). Cuestionario para la Evaluación de Metas Académicas en Secundaria (CEMA-II). Oviedo, España: Departamento de Psicología, Universidad de Oviedo.

Paris, S. G., Lipson, M. Y. y Wixson, K. K. (1983). Becoming a strategic reader. Contemporary Educational Psychology, 8(3), 293-316.

Pintrich, P.R. (1989). The dynamic interplay of student motivation and cognition in the college classroom. En C. Ames y M.L. Maehr (Eds.), Advances in motivation and achievement: Motivation-enhancing environments (Vol. 6, pp. 117-160). Greenwich, CT: JAI Press.

Pintrich, P. R. y De Groot, E. V. (1990). Motivational and self-regulated learning components of classroom academic performance. Journal of Educational Psychology, 82(1), 33.

Pintrich, P.R. y Schunk, D.H. (2002) Motivation in education: Theory, research and applications. New Jersey: Prentice-Hall.

Rodríguez, J. C. N., Cabanach, R. G., Piñeiro, I., Valle, A., Nuñez, J. y González Pienda, J. (2001). Metas de aproximación, metas de evitación y múltiples metas académicas. Psicothema, 13(4), 546-550.

Shibley J. y Durik, A.M. (2005). Gender, competence and motivation. En C. Dweck y A. Elliot (Eds.), Handbook of competence and motivation (pp. 375-391). New York: Guilford.

Steinmayr, R. y Spinarth, B. (2009) The importance of motivation as a predictor of school achievement. Learning and Individual Differences, 19, 80-90.

Valle, A., Cabanach, R., Rodríguez, S., Núñez, J., González-Pienda,J., y Rosário, P. (2007). Metas académicas y rendimiento en estudiantes de secundaria. Revista de Psicología General y Aplicada, 60, 181-192.

Valle, A., González, R., Cuevas, L. M., y Núñez, J. C. (1997). Patrones motivacionales en estudiantes universitarios: características diferenciales. Revista de Investigación Educativa, 15(1), 125-146.

Valle, A., Núñez, J., Cabanach, R., Rodríguez, S., González-Pienda, J. y Rosário, P. (2008). Capacidad predictiva de las metas académicas sobre el rendimiento en diferentes áreas curriculares. Revista Latinoamericana de Psicología, 40, 111-122.

Valle, A., Rodríguez, S., Cabanach, R., Núñez, J., González-Pienda,J. y Rosário, P. (2009). Metas académicas: Perspectiva histórica y conceptual e implicaciones educativas. Electronic Journal of Research in Educational Psychology, 7, 1073-1106. 